\title{
The Hahn Quantum Variational Calculus
}

\author{
A. B. Malinowska ${ }^{1} \quad$ D. F. M. Torres ${ }^{2}$ \\ (Submitted to JOTA: 3/March/2010; 4th revision: 9/June/2010; accepted: 18/June/2010)
}

\begin{abstract}
We introduce the Hahn quantum variational calculus. Necessary and sufficient optimality conditions for the basic, isoperimetric, and Hahn quantum Lagrange problems, are studied. We also show the validity of Leitmann's direct method for the Hahn quantum variational calculus, and give explicit solutions to some concrete problems. To illustrate the results, we provide several examples and discuss a quantum version of the well known Ramsey model of economics.
\end{abstract}

Keywords: Hahn's difference operator; Jackson-Norlünd's integral; quantum calculus; calculus of variations; Leitmann's principle; Ramsey model.

2010 Mathematics Subject Classification: 39A13; 39A70; 49J05; 49K05.

\section{Introduction}

Quantum difference operators are receiving an increase of interest due to their applications see, e.g., 1 15. Roughly speaking, a quantum calculus substitute the classical derivative by a difference operator, which allows to deal with sets of nondifferentiable functions. In [6], Hahn introduced the quantum difference operator $D_{q, \omega}$, where $\left.q \in\right] 0,1[$ and $\omega>0$ are fixed. The Hahn operator unifies (in the limit) the two most well known and used quantum difference operators: the Jackson $q$-difference derivative $D_{q}$, where $\left.q \in\right] 0,1\left[(\mathrm{cf}\right.$. [5, 7,, 8$]$ ); and the forward difference $\Delta_{\omega}$, where $\omega>0$ (cf. 9 11]). The Hahn difference operator is a successful tool for constructing families of orthogonal polynomials and investigating some approximation problems - see, e.g., [12 16. However, only in 2009 the construction of a proper inverse of $D_{q, \omega}$ and the associated integral calculus was given [17, 18.

In this work we introduce the variational Hahn calculus. More precisely, we formulate problems of the calculus of variations using Hahn's difference operator and the Jackson-Nörlund's integral. We discuss the fundamental concepts of a variational calculus, such as the Euler-Lagrange equations for the basic and isoperimetric problems, as well as Lagrange and optimal control problems. As particular cases we obtain the classical discrete-time calculus of variations [19, Chap. 8], the variational $q$-calculus [2,3, and the calculus of variations applied to Nörlund's sum [20].

The plan of the paper is as follows. In Sect. 2 some basic formulas of Hahn's difference operator and the associated Jackson-Nörlund integral calculus are briefly reviewed. Our results are formulated and proved in Sect. 3. Main results of the paper include necessary optimality conditions for the basic problem of calculus of variations (Theorem 3.2 in Sect. 3.1) and the isoperimetric

\footnotetext{
This work was partially supported by the Portuguese Foundation for Science and Technology (FCT) through the Center for Research and Development in Mathematics and Applications (CIDMA). The first author is currently a researcher at the University of Aveiro with the support of Bialystok University of Technology, via a project of the Polish Ministry of Science and Higher Education "Wsparcie miedzynarodowej mobilnosci naukowcow". The authors are grateful to Natália Martins for reading a preliminary version of the paper, and for many useful remarks.

${ }^{1}$ Assistant Professor in the Faculty of Computer Science, Białystok University of Technology, 15-351 Białystok, Poland. E-mail: abmalinowska@ua.pt

${ }^{2}$ Corresponding author. Associate Professor in the Department of Mathematics, University of Aveiro, 3810-193 Aveiro, Portugal. E-mail: delfim@ua.pt
} 
problem (Theorems 3.3 and 3.4 in Sect. 3.2), as well as a sufficient optimality condition for the basic problem (Theorem 3.5 in Sect. 3.3). In Sect. 3.4 we show that the direct method introduced by Leitmann in the sixties of the XX century [21, can also be applied with success to quantum variational problems via Hahn's difference operator and Jackson-Nörlund's integral. Leitmann's method is a venerable forty years old direct method that has shown through the times to be an universal and useful method in several different contexts - see, e.g., 2234 . Sect. 3.5 provides concrete examples of application of our results. Finally, in Sect. 3.6 we apply the developed Hahn variational calculus to obtain a quantum version of the well known Ramsey model and we finish the paper with conclusions in Sect. 4.

\section{Preliminaries}

Let $q \in] 0,1[$ and $\omega \in] 0,+\infty\left[\right.$ be given. Define $\omega_{0}:=\frac{\omega}{1-q}$. Throughout all the paper we assume $I$ to be an interval of $\mathbb{R}$ containing $\omega_{0}$.

Definition 2.1 (Hahn's difference operator). Let $f: I \rightarrow \mathbb{R}$. The Hahn difference operator is defined by

$$
D_{q, \omega} f(t):= \begin{cases}\frac{f(q t+\omega)-f(t)}{(q t+\omega)-t}, & \text { if } t \neq \omega_{0} \\ f^{\prime}(t), & \text { if } t=\omega_{0}\end{cases}
$$

provided that $f$ is differentiable at $\omega_{0}$ (we are using $f^{\prime}(t)$ to denote the Fréchet derivative). In this case, we call $D_{q, \omega} f$ the $q, \omega$-derivative of $f$ and say that $f$ is $q, \omega$-differentiable on $I$.

Example 2.1. Let $q \in] 0,1[, \omega=0$, and

$$
f(t)= \begin{cases}t^{2}, & \text { if } t \in \mathbb{Q} \\ -t^{2}, & \text { if } t \in \mathbb{R} \backslash \mathbb{Q} .\end{cases}
$$

In this case $\omega_{0}=0$, and $f$ is $q, \omega$-differentiable on the entire real line. However, $f$ is Fréchet differentiable in zero only.

Example 2.2. Let $q=\omega=1 / 2$. In this case $\omega_{0}=1$. It is easy to see that $f:[-1,1] \rightarrow \mathbb{R}$ given by

$$
f(t)= \begin{cases}-t, & \text { if } t \in(-1,0) \cup(0,1] \\ 0, & \text { if } t=-1 \\ 1, & \text { if } t=0\end{cases}
$$

is not a continuous function but is $q, \omega$-differentiable in $[-1,1]$ with

$$
D_{q, \omega} f(t)= \begin{cases}-1, & \text { if } t \in(-1,0) \cup(0,1] \\ 1, & \text { if } t=-1 \\ -3, & \text { if } t=0 .\end{cases}
$$

Note that,

$$
\lim _{q \uparrow 1} D_{q, \omega} f(t)=\Delta_{\omega} f(t), \quad \lim _{\omega \downarrow 0} D_{q, \omega} f(t)=D_{q} f(t), \quad \text { and } \lim _{\omega \downarrow 0, q \uparrow 1} D_{q, \omega} f(t)=f^{\prime}(t),
$$

where

$$
D_{q} f(t):=\frac{f(q t)-f(t)}{q t-t}, \quad t \neq 0,
$$

is the Jackson $q$-difference derivative [5, 7,8, and

$$
\Delta_{\omega} f(t):=\frac{f(t+\omega)-f(t)}{(t+\omega)-t}
$$

is the forward difference [911]. 
Remark 2.1. Let $\eta_{q, \omega}(t):=q t+\omega, t \in I$. Since $\left.q \in\right] 0,1[$ and $\omega \in] 0,+\infty\left[\right.$, then $\eta_{q, \omega}(t)$ is a contraction, $\eta_{q, \omega}(I) \subseteq I, \eta_{q, \omega}(t)<t$ for $t>\omega_{0}, \eta_{q, \omega}(t)>t$ for $t<\omega_{0}$, and $\eta_{q, \omega}\left(\omega_{0}\right)=\omega_{0}$.

The Hahn difference operator has the following properties:

Theorem 2.1 ( 17, 18]). (a) Let $f$ be $q, \omega$-differentiable on $I$ and $D_{q, \omega} f \equiv 0$ on $I$. Then $f$ is a constant. Conversely, $D_{q, \omega} c=0$ for any constant $c$.

(b) Let $f, g$ be $q, \omega$-differentiable at $t \in I$. Then,

(i) $D_{q, \omega}(f+g)(t)=D_{q, \omega} f(t)+D_{q, \omega} g(t)$,

(ii) $D_{q, \omega}(f g)(t)=D_{q, \omega}(f(t)) g(t)+f(q t+\omega) D_{q, \omega} g(t)$,

(iii) $D_{q, \omega}\left(\frac{f}{g}\right)(t)=\frac{D_{q, \omega}(f(t)) g(t)-f(t) D_{q, \omega} g(t)}{g(t) g(q t+\omega)}$ provided $g(t) g(q t+\omega) \neq 0$.

(c) $f(q t+\omega)=f(t)+((q t+\omega)-t) D_{q, \omega} f(t), t \in I$.

Example 2.3 ( [17, 18). Let $a, b \in \mathbb{R}$. We have

$$
D_{q, \omega}(a t+b)^{n}=a \sum_{k=0}^{n-1}(a(q t+\omega)+b)^{k}(a t+b)^{n-k-1},
$$

for $n \in \mathbb{N}$ and $t \neq \omega_{0}$.

Following [17, 18, 35, we define the inverse of the operator $D_{q, \omega}$ :

Definition 2.2. Let $I$ be a closed interval of $\mathbb{R}$ such that $\omega_{0}, a, b \in I$. For $f: I \rightarrow \mathbb{R}$ we define the $q$, $\omega$-integral of $f$ from $a$ to $b$ by

$$
\int_{a}^{b} f(t) d_{q, \omega} t:=\int_{\omega_{0}}^{b} f(t) d_{q, \omega} t-\int_{\omega_{0}}^{a} f(t) d_{q, \omega} t
$$

where

$$
\int_{\omega_{0}}^{x} f(t) d_{q, \omega} t:=(x(1-q)-\omega) \sum_{k=0}^{\infty} q^{k} f\left(x q^{k}+[k]_{q, \omega}\right), \quad x \in I,
$$

with $[k]_{q, \omega}:=\frac{\omega\left(1-q^{k}\right)}{1-q}$ for $k \in \mathbb{N}_{0}=\mathbb{N} \cup\{0\}$, provided that the series converges at $x=a$ and $x=b$. In this case, $f$ is called $q, \omega$-integrable on $[a, b]$. We say that $f$ is $q, \omega$-integrable over $I$ iff it is $q, \omega$-integrable over $[a, b]$, for all $a, b \in I$.

Note that, in the integral formulas (2) and (3), when $\omega \downarrow 0$ we obtain the Jackson $q$-integral

$$
\int_{a}^{b} f(t) d_{q} t=\int_{0}^{b} f(t) d_{q} t-\int_{0}^{a} f(t) d_{q} t
$$

where

$$
\int_{0}^{x} f(t) d_{q} t=x(1-q) \sum_{k=0}^{\infty} q^{k} f\left(x q^{k}\right)
$$

(see, e.g., [36]); while if $q \uparrow 1$ we obtain the Nörlund sum

$$
\int_{a}^{b} f(t) \Delta_{\omega} t=\int_{+\infty}^{b} f(t) \Delta_{\omega} t-\int_{+\infty}^{a} f(t) \Delta_{\omega} t
$$

where

$$
\int_{+\infty}^{x} f(t) \Delta_{\omega} t=-\omega \sum_{k=0}^{+\infty} f(x+k \omega)
$$

(see, e.g., [10,37,38]). This is why the integral defined by (22) and (3) is called the Jackson-Nörlund integral.

If $f: I \rightarrow \mathbb{R}$ is continuous at $\omega_{0}$, then $f$ is $q, \omega$-integrable over $I$ (see [17, 18] for the proof). 
Theorem $2.2([17,18])$. Assume $f: I \rightarrow \mathbb{R}$ be continuous at $\omega_{0}$. Define

$$
F(x):=\int_{\omega_{0}}^{x} f(t) d_{q, \omega} t
$$

Then $F$ is continuous at $\omega_{0}$. Furthermore, $D_{q, \omega} F(x)$ exists for every $x \in I$ and $D_{q, \omega} F(x)=f(x)$. Conversely,

$$
\int_{a}^{b} D_{q, \omega} f(t) d_{q, \omega} t=f(b)-f(a)
$$

for all $a, b \in I$.

The $q, \omega$-integral has the following properties.

Theorem 2.3 ( [17, 18]). (a) Let $f, g: I \rightarrow \mathbb{R}$ be $q, \omega$-integrable on $I, a, b, c \in I$ and $k \in \mathbb{R}$. Then,

(i) $\int_{a}^{a} f(t) d_{q, \omega} t=0$,

(ii) $\int_{a}^{b} k f(t) d_{q, \omega} t=k \int_{a}^{b} f(t) d_{q, \omega} t$,

(iii) $\int_{a}^{b} f(t) d_{q, \omega} t=-\int_{b}^{a} f(t) d_{q, \omega} t$,

(iv) $\int_{a}^{b} f(t) d_{q, \omega} t=\int_{a}^{c} f(t) d_{q, \omega} t+\int_{c}^{b} f(t) d_{q, \omega} t$,

(v) $\int_{a}^{b}(f(t)+g(t)) d_{q, \omega} t=\int_{a}^{b} f(t) d_{q, \omega} t+\int_{a}^{b} g(t) d_{q, \omega} t$.

(b) Every Riemann integrable function $f$ on $I$ is $q$, $\omega$-integrable on $I$.

(c) If $f, g: I \rightarrow \mathbb{R}$ are continuous at $\omega_{0}$, then

$$
\int_{a}^{b} f(t) D_{q, \omega} g(t) d_{q, \omega} t=\left.f(t) g(t)\right|_{t=a} ^{t=b}-\int_{a}^{b} D_{q, \omega}(f(t)) g(q t+\omega) d_{q, \omega} t, \quad a, b \in I .
$$

Property (c) in Theorem 2.3 is the integration by parts formula for the Jackson-Nörlund integral, and will be useful in the proof of our Theorem 3.2

Lemma 2.1 ( [17, 18]). Let $s \in I, f$ and $g$ be $q, \omega$-integrable. If $|f(t)| \leq g(t)$ for all $t \in\left\{q^{n} s+\right.$ $\left.[n]_{q, \omega}: n \in \mathbb{N}_{0}\right\}, s \in I$, then

$$
\left|\int_{\omega_{0}}^{b} f(t) d_{q, \omega} t\right| \leq \int_{\omega_{0}}^{b} g(t) d_{q, \omega} t, \quad\left|\int_{a}^{b} f(t) d_{q, \omega} t\right| \leq \int_{a}^{b} g(t) d_{q, \omega} t
$$

for all $a, b \in\left\{q^{n} s+[n]_{q, \omega}: n \in \mathbb{N}_{0}\right\}$.

However, it should be noted that the inequality

$$
\left|\int_{a}^{b} f(t) d_{q, \omega} t\right| \leq \int_{a}^{b}|f(t)| d_{q, \omega} t
$$

$a, b \in I$, is not always true. For an example we refer the reader to [17, 18.

\section{Main Results}

Let $a, b \in I$ with $a<b$. We define the $q, \omega$-interval by

$$
[a, b]_{q, \omega}:=\left\{q^{n} a+[n]_{q, \omega}: n \in \mathbb{N}_{0}\right\} \cup\left\{q^{n} b+[n]_{q, \omega}: n \in \mathbb{N}_{0}\right\} \cup\left\{\omega_{0}\right\} .
$$

By $\mathcal{D}$ we denote the set of all real valued functions defined on $[a, b]_{q, \omega}$ and continuous at $\omega_{0}$. 
Lemma 3.1 (Fundamental Lemma of the Hahn quantum variational calculus). Let $f \in \mathcal{D}$. One has $\int_{a}^{b} f(t) h(q t+\omega) d_{q, \omega} t=0$ for all functions $h \in \mathcal{D}$ with $h(a)=h(b)=0$ if and only if $f(t)=0$ for all $t \in[a, b]_{q, \omega}$.

Proof. The implication " $\Leftarrow$ " is obvious. Let us prove the implication " $\Rightarrow$ ". Suppose, by contradiction, that $f(p) \neq 0$ for some $p \in[a, b]_{q, \omega}$.

Case I. If $p \neq \omega_{0}$, then $p=q^{k} a+[k]_{q, \omega}$ or $p=q^{k} b+[k]_{q, \omega}$ for some $k \in \mathbb{N}_{0}$. Observe that $a(1-q)-\omega$ and $b(1-q)-\omega$ cannot vanish simultaneously. Therefore, without loss of generality, we can assume $a(1-q)-\omega \neq 0$ and $p=q^{k} a+[k]_{q, \omega}$. Define

$$
h(t)= \begin{cases}f\left(q^{k} a+[k]_{q, \omega}\right), & \text { if } t=q^{k+1} a+[k+1]_{q, \omega} \\ 0, & \text { otherwise. }\end{cases}
$$

Then,

$$
\int_{a}^{b} f(t) h(q t+\omega) d_{q, \omega} t=-(a(1-q)-\omega) q^{k} f\left(q^{k} a+[k]_{q, \omega}\right) h\left(q^{k+1} a+[k+1]_{q, \omega}\right) \neq 0,
$$

which is a contradiction.

Case II. If $p=\omega_{0}$, then without loss of generality we can assume $f\left(\omega_{0}\right)>0$. We know that (see 17, 18, for more details)

$$
\lim _{n \uparrow \infty} q^{n} a+[n]_{q, \omega}=\lim _{n \uparrow \infty} q^{n} b+[n]_{q, \omega}=\omega_{0} .
$$

As $f$ is continuous at $\omega_{0}$, we have

$$
\lim _{n \uparrow \infty} f\left(q^{n} a+[n]_{q, \omega}\right)=\lim _{n \uparrow \infty} f\left(q^{n} b+[n]_{q, \omega}\right)=f\left(\omega_{0}\right) .
$$

Therefore, there exists $N \in \mathbb{N}$, such that for all $n>N$ the inequalities

$$
f\left(q^{n} a+[n]_{q, \omega}\right)>0 \quad \text { and } \quad f\left(q^{n} b+[n]_{q, \omega}\right)>0
$$

hold. If $\omega_{0} \neq a, b$, then we define

$$
h(t)=\left\{\begin{array}{lll}
f\left(q^{n} b+[n]_{q, \omega}\right), & \text { if } t=q^{n+1} a+[n+1]_{q, \omega}, & \text { for all } n>N \\
f\left(q^{n} a+[n]_{q, \omega}\right), & \text { if } t=q^{n+1} b+[n+1]_{q, \omega}, & \text { for all } n>N \\
0, & \text { otherwise }
\end{array}\right.
$$

Hence,

$$
\int_{a}^{b} f(t) h(q t+\omega) d_{q, \omega} t=(b-a)(1-q) \sum_{n=N}^{\infty} q^{n} f\left(q^{n} a+[n]_{q, \omega}\right) f\left(q^{n} b+[n]_{q, \omega}\right) \neq 0,
$$

which is a contradiction. If $\omega_{0}=b$, then we define

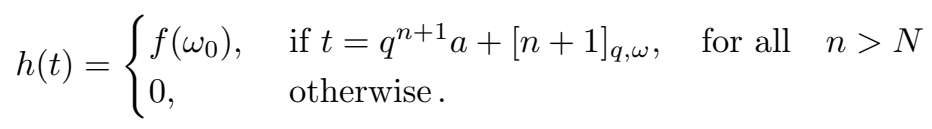

Hence,

$$
\int_{a}^{b} f(t) h(q t+\omega) d_{q, \omega} t=-\int_{\omega_{0}}^{a} f(t) h(q t+\omega) d_{q, \omega} t=-(a(1-q)-\omega) \sum_{n=N}^{\infty} q^{n} f\left(q^{n} a+[n]_{q, \omega}\right) f\left(\omega_{0}\right) \neq 0,
$$

which is a contradiction. Similarly, we show the case when $\omega_{0}=a$. 
Let $\mathbb{E}$ be the linear space of functions $y \in \mathcal{D}$ for which the $q, \omega$-derivative is bounded on $[a, b]_{q, \omega}$ and continuous at $\omega_{0}$. We equip $\mathbb{E}$ with the norm

$$
\|y\|_{1}=\sup _{t \in[a, b]_{q, \omega}}|y(t)|+\sup _{t \in[a, b]_{q, \omega}}\left|D_{q, \omega} y(t)\right| .
$$

For $s \in I$ we set

$$
[s]_{q, \omega}=\left\{q^{n} s+[n]_{q, \omega}: n \in \mathbb{N}_{0}\right\} \cup\left\{\omega_{0}\right\} .
$$

In the sequel we need one more result. The following definition and lemma are similar to 39].

Definition 3.1. Let $\left.g:[s]_{q, \omega} \times\right]-\bar{\theta}, \bar{\theta}\left[\rightarrow \mathbb{R}\right.$. We say that $g(t, \cdot)$ is continuous in $\theta_{0}$, uniformly in $t$, iff for every $\varepsilon>0$ there exists $\delta>0$ such that $\left|\theta-\theta_{0}\right|<\delta$ implies $\left|g(t, \theta)-g\left(t, \theta_{0}\right)\right|<\varepsilon$ for all $t \in[s]_{q, \omega}$. Furthermore, we say that $g(t, \cdot)$ is differentiable at $\theta_{0}$, uniformly in $t$, iff for every $\varepsilon>0$ there exists $\delta>0$ such that $0<\left|\theta-\theta_{0}\right|<\delta$ implies

$$
\left|\frac{g(t, \theta)-g\left(t, \theta_{0}\right)}{\theta-\theta_{0}}-\partial_{2} g\left(t, \theta_{0}\right)\right|<\varepsilon,
$$

where $\partial_{2} g=\frac{\partial g}{\partial \theta}$ for all $t \in[s]_{q, \omega}$.

Lemma 3.2. Assume $g(t, \cdot)$ be differentiable at $\theta_{0}$, uniformly in $t$ in $[s]_{q, \omega}$, and that $G(\theta):=$ $\int_{\omega_{0}}^{s} g(t, \theta) d_{q, \omega} t$, for $\theta$ near $\theta_{0}$, and $\int_{\omega_{0}}^{s} \partial_{2} g\left(t, \theta_{0}\right) d_{q, \omega}$ exist. Then, $G(\theta)$ is differentiable at $\theta_{0}$ with $G^{\prime}\left(\theta_{0}\right)=\int_{\omega_{0}}^{s} \partial_{2} g\left(t, \theta_{0}\right) d_{q, \omega} t$.

Proof. Let $\varepsilon>0$ be arbitrary. Since $g(t, \cdot)$ is differentiable at $\theta_{0}$, uniformly in $t$, there exists $\delta>0$, such that, for all $t \in[s]_{q, \omega}$, and for $0<\left|\theta-\theta_{0}\right|<\delta$, the following inequality holds:

$$
\left|\frac{g(t, \theta)-g\left(t, \theta_{0}\right)}{\theta-\theta_{0}}-\partial_{2} g\left(t, \theta_{0}\right)\right|<\frac{\varepsilon}{s-\omega_{0}} .
$$

Applying Theorem 2.3 and Lemma 2.1, for $0<\left|\theta-\theta_{0}\right|<\delta$, we have

$$
\begin{aligned}
& \left|\frac{G(\theta)-G\left(\theta_{0}\right)}{\theta-\theta_{0}}-G^{\prime}\left(\theta_{0}\right)\right|=\left|\frac{\int_{\omega_{0}}^{s} g(t, \theta) d_{q, \omega} t-\int_{\omega_{0}}^{s} g\left(t, \theta_{0}\right) d_{q, \omega} t}{\theta-\theta_{0}}-\int_{\omega_{0}}^{s} \partial_{2} g\left(t, \theta_{0}\right) d_{q, \omega} t\right| \\
& =\left|\int_{\omega_{0}}^{s}\left[\frac{g(t, \theta)-g\left(t, \theta_{0}\right)}{\theta-\theta_{0}}-\partial_{2} g\left(t, \theta_{0}\right)\right] d_{q, \omega} t\right|<\int_{\omega_{0}}^{s} \frac{\varepsilon}{s-\omega_{0}} d_{q, \omega} t=\frac{\varepsilon}{s-\omega_{0}} \int_{\omega_{0}}^{s} 1 d_{q, \omega} t=\varepsilon .
\end{aligned}
$$

Hence, $G(\cdot)$ is differentiable at $\theta_{0}$ and $G^{\prime}\left(\theta_{0}\right)=\int_{\omega_{0}}^{s} \partial_{2} g\left(t, \theta_{0}\right) d_{q, \omega} t$.

\subsection{The Hahn Quantum Euler-Lagrange Equation}

We consider the variational problem of finding minima (or maxima) of a functional

$$
\mathcal{L}[y]=\int_{a}^{b} f\left(t, y(q t+\omega), D_{q, \omega} y(t)\right) d_{q, \omega} t
$$

over all $y \in \mathbb{E}$ satisfying the boundary conditions

$$
y(a)=\alpha, \quad y(b)=\beta, \quad \alpha, \beta \in \mathbb{R},
$$

where $f:[a, b]_{q, \omega} \times \mathbb{R} \times \mathbb{R} \rightarrow \mathbb{R}$ is a given function. A function $y \in \mathbb{E}$ is said to be admissible iff it satisfies the endpoint conditions (6). Let us denote by $\partial_{2} f$ and $\partial_{3} f$, respectively, the partial derivatives of $f(\cdot, \cdot, \cdot)$ with respect to its second and third argument. In the sequel, we assume that $(u, v) \rightarrow f(t, u, v)$ be a $C^{1}\left(\mathbb{R}^{2}, \mathbb{R}\right)$ function for any $t \in[a, b]_{q, \omega}$, and $f\left(\cdot, y(\cdot), D_{q, \omega} y(\cdot)\right)$, $\partial_{2} f\left(\cdot, y(\cdot), D_{q, \omega} y(\cdot)\right)$, and $\partial_{3} f\left(\cdot, y(\cdot), D_{q, \omega} y(\cdot)\right)$ are continuous at $\omega_{0}$ for any admissible function $y(\cdot)$. Finally, an $h \in \mathbb{E}$ is called an admissible variation provided $h(a)=h(b)=0$. 
For an admissible variation $h$, we define function $\phi:]-\bar{\varepsilon}, \bar{\varepsilon}[\rightarrow \mathbb{R}$ by

$$
\phi(\varepsilon)=\phi(\varepsilon ; y, h):=\mathcal{L}[y+\varepsilon h] .
$$

The first variation of problem (5) - (6) is defined by

$$
\delta \mathcal{L}[y, h]:=\phi(0 ; y, h)=\phi^{\prime}(0) .
$$

Observe that,

$$
\begin{aligned}
\mathcal{L}[y+\varepsilon h]= & \int_{a}^{b} f\left(t, y(q t+\omega)+\varepsilon h(q t+\omega), D_{q, \omega} y(t)+\varepsilon D_{q, \omega} h(t)\right) d_{q, \omega} t \\
= & \int_{\omega_{0}}^{b} f\left(t, y(q t+\omega)+\varepsilon h(q t+\omega), D_{q, \omega} y(t)+\varepsilon D_{q, \omega} h(t)\right) d_{q, \omega} t \\
& \quad-\int_{\omega_{0}}^{a} f\left(t, y(q t+\omega)+\varepsilon h(q t+\omega), D_{q, \omega} y(t)+\varepsilon D_{q, \omega} h(t)\right) d_{q, \omega} t .
\end{aligned}
$$

Writing

$$
\mathcal{L}_{b}[y+\varepsilon h]=\int_{\omega_{0}}^{b} f\left(t, y(q t+\omega)+\varepsilon h(q t+\omega), D_{q, \omega} y(t)+\varepsilon D_{q, \omega} h(t)\right) d_{q, \omega} t
$$

and

$$
\mathcal{L}_{a}[y+\varepsilon h]=\int_{\omega_{0}}^{a} f\left(t, y(q t+\omega)+\varepsilon h(q t+\omega), D_{q, \omega} y(t)+\varepsilon D_{q, \omega} h(t)\right) d_{q, \omega} t
$$

we have

$$
\mathcal{L}[y+\varepsilon h]=\mathcal{L}_{b}[y+\varepsilon h]-\mathcal{L}_{a}[y+\varepsilon h] .
$$

Therefore,

$$
\delta \mathcal{L}[y, h]=\delta \mathcal{L}_{b}[y, h]-\delta \mathcal{L}_{a}[y, h] .
$$

Knowing (7), the following lemma is a direct consequence of Lemma 3.2,

Lemma 3.3. Put $g(t, \varepsilon)=f\left(t, y(q t+\omega)+\varepsilon h(q t+\omega), D_{q, \omega} y(t)+\varepsilon D_{q, \omega} h(t)\right)$ for $\left.\varepsilon \in\right]-\bar{\varepsilon}, \bar{\varepsilon}[$. Assume that:

(i) $g(t, \cdot)$ be differentiable at 0 , uniformly in $t \in[a]_{q, \omega}$, and $g(t, \cdot)$ be differentiable at 0 , uniformly in $t \in[b]_{q, \omega}$;

(ii) $\mathcal{L}_{a}[y+\varepsilon h]$ and $\mathcal{L}_{b}[y+\varepsilon h]$, for $\varepsilon$ near 0 , exist;

(iii) $\int_{\omega_{0}}^{a} \partial_{2} g(t, 0) d_{q, \omega} t$ and $\int_{\omega_{0}}^{b} \partial_{2} g(t, 0) d_{q, \omega} t$ exist.

Then,

$$
\delta \mathcal{L}[y, h]=\int_{a}^{b}\left[\partial_{2} f\left(t, y(q t+\omega), D_{q, \omega} y(t)\right) h(q t+\omega)+\partial_{3} f\left(t, y(q t+\omega), D_{q, \omega} y(t)\right) D_{q, \omega} h(t)\right] d_{q, \omega} t .
$$

In the sequel, we always assume, without mentioning it explicitly, that variational problems satisfy the assumptions of Lemma 3.3 .

Definition 3.2. An admissible function $\tilde{y}$ is said to be a local minimizer (resp. a local maximizer) to problem (5) -(6) iff there exists $\delta>0$, such that $\mathcal{L}[\tilde{y}] \leq \mathcal{L}[y]($ resp. $\mathcal{L}[\tilde{y}] \geq \mathcal{L}[y])$ for all admissible $y$ with $\|y-\tilde{y}\|_{1}<\delta$.

The following result offers a necessary condition for local extremizer.

Theorem 3.1 (A necessary optimality condition for problem (5)-(6)). Suppose that the optimal path to problem (5) (6) exists and is given by $\tilde{y}$. Then, $\delta \mathcal{L}[\tilde{y}, h]=0$. 
Proof. Without loss of generality, we can assume $\tilde{y}$ to be a local minimizer. Let $h$ be any admissible variation and define a function $\phi:]-\bar{\varepsilon}, \bar{\varepsilon}[\rightarrow \mathbb{R}$ by $\phi(\varepsilon)=\mathcal{L}[\tilde{y}+\varepsilon h]$. Since $\tilde{y}$ is a local minimizer, there exists $\delta>0$, such that $\mathcal{L}[\tilde{y}] \leq \mathcal{L}[y]$ for all admissible $y$ with $\|y-\tilde{y}\|_{1}<\delta$. Therefore, $\phi(\varepsilon)=\mathcal{L}[\tilde{y}+\varepsilon h] \geq \mathcal{L}[\tilde{y}]=\phi(0)$ for all $\varepsilon<\frac{\delta}{\|h\|_{1}}$. Hence, $\phi$ has a local minimum at $\varepsilon=0$, and thus our assertion follows.

Theorem 3.2 (The Hahn quantum Euler-Lagrange equation for problem (5)-(6)). Suppose that the optimal path to problem (5) -(6) exists and is given by $\tilde{y}$. Then,

$$
D_{q, \omega} \partial_{3} f\left(t, \tilde{y}(q t+\omega), D_{q, \omega} \tilde{y}(t)\right)=\partial_{2} f\left(t, \tilde{y}(q t+\omega), D_{q, \omega} \tilde{y}(t)\right)
$$

for all $t \in[a, b]_{q, \omega}$.

Proof. Suppose that $\mathcal{L}$ has a local extremum at $\tilde{y}$. Let $h$ be any admissible variation and define a function $\phi:]-\bar{\varepsilon}, \bar{\varepsilon}[\rightarrow \mathbb{R}$ by $\phi(\varepsilon)=\mathcal{L}[\tilde{y}+\varepsilon h]$. By Theorem [3.1, a necessary condition for $\tilde{y}$ to be an extremizer is given by

$$
\left.\phi^{\prime}(\varepsilon)\right|_{\varepsilon=0}=0 \Leftrightarrow \int_{a}^{b}\left[\partial_{2} f(\cdots) h(q t+\omega)+\partial_{3} f(\cdots) D_{q, \omega} h(t)\right] d_{q, \omega} t=0,
$$

where $(\cdots)=\left(t, \tilde{y}(q t+\omega), D_{q, \omega} \tilde{y}(t)\right)$. Integration by parts (see item (c) in Theorem 2.3) gives

$$
\int_{a}^{b} \partial_{3} f(\cdots) D_{q, \omega} h(t) d_{q, \omega} t=\left.\partial_{3} f(\cdots) h(t)\right|_{t=a} ^{t=b}-\int_{a}^{b} D_{q, \omega} \partial_{3} f(\cdots) h(q t+\omega) d_{q, \omega} t .
$$

Because $h(a)=h(b)=0$, the necessary condition (9) can be written as

$$
0=\int_{a}^{b}\left(\partial_{2} f(\cdots)-D_{q, \omega} \partial_{3} f(\cdots)\right) h(q t+\omega) d_{q, \omega} t
$$

for all $h$ such that $h(a)=h(b)=0$. Thus, by Lemma 3.1, we have

$$
\partial_{2} f(\cdots)-D_{q, \omega} \partial_{3} f(\cdots)=0
$$

for all $t \in[a, b]_{q, \omega}$.

Remark 3.1. If the function $f$ under the sign of integration (the Lagrangian) is given by $f=$ $f\left(t, y_{1}, \ldots, y_{n}, D_{q, \omega} y_{1}, \ldots, D_{q, \omega} y_{n}\right)$, then the necessary optimality condition is given by $n$ equations similar to (8), one equation for each variable.

\subsection{The Hahn Quantum Isoperimetric Problem}

Let us consider now the isoperimetric problem, which consists of minimizing or maximizing

$$
\mathcal{L}[y]=\int_{a}^{b} f\left(t, y(q t+\omega), D_{q, \omega} y(t)\right) d_{q, \omega} t
$$

over all $y \in \mathbb{E}$ satisfying the boundary conditions

$$
y(a)=\alpha, \quad y(b)=\beta,
$$

and the constraint

$$
\mathcal{K}[y]=\int_{a}^{b} g\left(t, y(q t+\omega), D_{q, \omega} y(t)\right) d_{q, \omega} t=k,
$$

where $\alpha, \beta$, and $k$ are given real numbers. We assume that:

(i) $(u, v) \rightarrow f(t, u, v)$ and $(u, v) \rightarrow g(t, u, v)$ be $C^{1}\left(\mathbb{R}^{2}, \mathbb{R}\right)$ functions for any $t \in[a, b]_{q, \omega}$; 
(ii) functions $f\left(\cdot, y(\cdot) D_{q, \omega} y(\cdot)\right), \partial_{2} f\left(\cdot, y(\cdot), D_{q, \omega} y(\cdot)\right)$ and $\partial_{3} f\left(\cdot, y(\cdot), D_{q, \omega} y(\cdot)\right), g\left(\cdot, y(\cdot), D_{q, \omega} y(\cdot)\right)$, $\partial_{2} g\left(\cdot, y(\cdot), D_{q, \omega} y(\cdot)\right)$ and $\partial_{3} g\left(\cdot, y(\cdot), D_{q, \omega} y(\cdot)\right)$, be continuous at $\omega_{0}$ for any admissible function $y(\cdot)$.

Definition 3.3. An admissible function $\tilde{y}$ is said to be a local minimizer (resp. local maximizer) for the isoperimetric problem (10)-(12) iff there exists $\delta>0$ such that $\mathcal{L}[\tilde{y}] \leq \mathcal{L}[y]($ resp. $\mathcal{L}[\tilde{y}] \geq \mathcal{L}[y])$ for all admissible $y$ satisfying the boundary conditions (11), the isoperimetric constraint (12), and $\|y-\tilde{y}\|_{1}<\delta$.

Definition 3.4. We say that $\tilde{y}$ is an extremal for $\mathcal{K}$, iff (8) holds with respect to (12):

$$
D_{q, \omega} \partial_{3} g\left(t, \tilde{y}(q t+\omega), D_{q, \omega} \tilde{y}(t)\right)=\partial_{2} g\left(t, \tilde{y}(q t+\omega), D_{q, \omega} \tilde{y}(t)\right)
$$

for all $t \in[a, b]_{q, \omega}$. An extremizer (i.e., a local minimizer or a local maximizer) for the problem (10)-(12), that is not an extremal for $\mathcal{K}$, is said to be a normal extremizer; otherwise (i.e., if it is an extremal for $\mathcal{K}$ ), the extremizer is said to be abnormal.

Theorem 3.3. If $\tilde{y}$ is a normal extremizer for the isoperimetric problem (10)-(12), then there exists a real $\lambda$, such that

$$
D_{q, \omega} \partial_{3} F\left(t, \tilde{y}(q t+\omega), D_{q, \omega} \tilde{y}(t)\right)=\partial_{2} F\left(t, \tilde{y}(q t+\omega), D_{q, \omega} \tilde{y}(t)\right)
$$

for all $t \in[a, b]_{q, \omega}$, where $F=f-\lambda g$.

Proof. Consider a variation of $\tilde{y}$, say $\bar{y}=\tilde{y}+\varepsilon_{1} h_{1}+\varepsilon_{2} h_{2}$, where $h_{i} \in \mathbb{E}, h_{i}(a)=h_{i}(b)=0$, and $\varepsilon_{i}$ is a sufficiently small parameter, $i=1,2$. Here, $h_{1}$ is an arbitrary fixed function and $h_{2}$ is a fixed function that will be chosen later. Define

$$
\bar{K}\left(\varepsilon_{1}, \varepsilon_{2}\right)=\mathcal{K}[\bar{y}]=\int_{a}^{b} g\left(t, \bar{y}(q t+\omega), D_{q, \omega} \bar{y}(t)\right) d_{q, \omega} t-k .
$$

We have

$$
\left.\frac{\partial \bar{K}}{\partial \varepsilon_{2}}\right|_{(0,0)}=\int_{a}^{b}\left[\partial_{2} g(\cdots) h_{2}(q t+\omega)+\partial_{3} g(\cdots) D_{q, \omega} h_{2}(t)\right] d_{q, \omega} t
$$

where $(\cdots)=\left(t, \tilde{y}(q t+\omega), D_{q, \omega} \tilde{y}(t)\right)$. Integration by parts gives

$$
\left.\frac{\partial \bar{K}}{\partial \varepsilon_{2}}\right|_{(0,0)}=\int_{a}^{b}\left(\partial_{2} g(\cdots)-D_{q, \omega} \partial_{3} g(\cdots)\right) h_{2}(q t+\omega) d_{q, \omega} t
$$

since $h_{2}(a)=h_{2}(b)=0$. By Lemma 3.1, there exists $h_{2}$ such that $\left.\frac{\partial \bar{K}}{\partial \varepsilon_{2}}\right|_{(0,0)} \neq 0$. Since $\bar{K}(0,0)=0$, by the Dini-Ljusternik implicit function theorem we conclude that there exists a function $\varepsilon_{2}$, defined in the neighborhood of zero, such that $\bar{K}\left(\varepsilon_{1}, \varepsilon_{2}\left(\varepsilon_{1}\right)\right)=0$, i.e., we may choose a subset of variations $\bar{y}$ satisfying the isoperimetric constraint.

Let us now consider the real function

$$
\bar{L}\left(\varepsilon_{1}, \varepsilon_{2}\right)=\mathcal{L}[\bar{y}]=\int_{a}^{b} f\left(t, \bar{y}(q t+\omega), D_{q, \omega} \bar{y}(t)\right) d_{q, \omega} t .
$$

By hypothesis, $(0,0)$ is an extremal of $\bar{L}$ subject to the constraint $\bar{K}=0$ and $\nabla \bar{K}(0,0) \neq \mathbf{0}$. By the Lagrange multiplier rule, there exists some real $\lambda$, such that $\nabla(\bar{L}(0,0)-\lambda \bar{K}(0,0))=\mathbf{0}$. Having in mind that $h_{1}(a)=h_{1}(b)=0$, we can write

$$
\left.\frac{\partial \bar{L}}{\partial \varepsilon_{1}}\right|_{(0,0)}=\int_{a}^{b}\left(\partial_{2} f(\cdots)-D_{q, \omega} \partial_{3} f(\cdots)\right) h_{1}(q t+\omega) d_{q, \omega} t
$$

and

$$
\left.\frac{\partial \bar{K}}{\partial \varepsilon_{1}}\right|_{(0,0)}=\int_{a}^{b}\left(\partial_{2} g(\cdots)-D_{q, \omega} \partial_{3} g(\cdots)\right) h_{1}(q t+\omega) d_{q, \omega} t .
$$


Therefore,

$$
\int_{a}^{b}\left[\left(\partial_{2} f(\cdots)-D_{q, \omega} \partial_{3} f(\cdots)\right)-\lambda\left(\partial_{2} g(\cdots)-D_{q, \omega} \partial_{3} g(\cdots)\right)\right] h_{1}(q t+\omega) d_{q, \omega} t=0 .
$$

As (14) holds for any $h_{1}$, by Lemma 3.1 we have

$$
\partial_{2} f(\cdots)-D_{q, \omega} \partial_{3} f(\cdots)-\lambda\left(\partial_{2} g(\cdots)-D_{q, \omega} \partial_{3} g(\cdots)\right)=0 .
$$

We get (13) by writing $F=f-\lambda g$.

One can easily cover abnormal extremizers within our result by introducing an extra multiplier $\lambda_{0}$ associated with the cost functional.

Theorem 3.4. If $\tilde{y}$ is an extremizer for the isoperimetric problem (10)-(12), then there exist two constants $\lambda_{0}$ and $\lambda$, not both zero, such that

$$
D_{q, \omega} \partial_{3} F\left(t, \tilde{y}(q t+\omega), D_{q, \omega} \tilde{y}(t)\right)=\partial_{2} F\left(t, \tilde{y}(q t+\omega), D_{q, \omega} \tilde{y}(t)\right)
$$

for all $t \in[a, b]_{q, \omega}$, where $F=\lambda_{0} f-\lambda g$.

Proof. Following the proof of Theorem 3.3 since $(0,0)$ is an extremal of $\bar{L}$ subject to the constraint $\bar{K}=0$, the extended Lagrange multiplier rule (see, for instance, [40, Theorem 4.1.3]) asserts the existence of reals $\lambda_{0}$ and $\lambda$, not both zero, such that $\nabla\left(\lambda_{0} \bar{L}(0,0)-\lambda \bar{K}(0,0)\right)=\mathbf{0}$. Therefore,

$$
\begin{gathered}
\left.\lambda_{0} \frac{\partial \bar{L}}{\partial \varepsilon_{1}}\right|_{(0,0)}-\left.\lambda \frac{\partial \bar{K}}{\partial \varepsilon_{1}}\right|_{(0,0)}=0 \\
\Leftrightarrow \int_{a}^{b}\left[\lambda_{0}\left(\partial_{2} f(\cdots)-D_{q, \omega} \partial_{3} f(\cdots)\right)-\lambda\left(\partial_{2} g(\cdots)-D_{q, \omega} \partial_{3} g(\cdots)\right)\right] h_{1}(q t+\omega) d_{q, \omega} t=0 .
\end{gathered}
$$

Since (16) holds for any $h_{1}$, it follows by Lemma 3.1 that

$$
\lambda_{0}\left(\partial_{2} f(\cdots)-D_{q, \omega} \partial_{3} f(\cdots)\right)-\lambda\left(\partial_{2} g(\cdots)-D_{q, \omega} \partial_{3} g(\cdots)\right)=0 .
$$

The desired condition (15) follows by taking $F=\lambda_{0} f-\lambda g$.

Remark 3.2. If $\tilde{y}$ is a normal extremizer for the isoperimetric problem (10)-(12), then we can choose $\lambda_{0}=1$ in Theorem 3.4 and obtain Theorem 3.3. For abnormal extremizers, Theorem 3.4 holds with $\lambda_{0}=0$. The condition $\left(\lambda_{0}, \lambda\right) \neq \mathbf{0}$ guarantees that Theorem 3.4 is a nontrivial necessary condition. In general we cannot guarantee, a priori, that $\lambda_{0}$ be different from zero. The interested reader about abnormality is referred to the book 41 .

Suppose now that it is required to find functions $y_{1}$ and $y_{2}$ for which the functional

$$
\mathcal{L}\left[y_{1}, y_{2}\right]=\int_{a}^{b} f\left(t, y_{1}(q t+\omega), y_{2}(q t+\omega), D_{q, \omega} y_{1}(t), D_{q, \omega} y_{2}(t)\right) d_{q, \omega} t
$$

has an extremum, where the admissible functions satisfy the boundary conditions

$$
\left(y_{1}(a), y_{2}(a)\right)=\left(y_{1}^{a}, y_{2}^{a}\right) \text { and }\left(y_{1}(b), y_{2}(b)\right)=\left(y_{1}^{b}, y_{2}^{b}\right)
$$

and the subsidiary nonholonomic condition

$$
g\left(t, y_{1}(q t+\omega), y_{2}(q t+\omega), D_{q, \omega} y_{1}(t), D_{q, \omega} y_{2}(t)\right)=0 .
$$

The problem (17)-(19) can be reduced to the isoperimetric one by transforming (19) into a constraint of the type (12). For that, we multiply both sides of (19) by an arbitrary function $\lambda(t)$, and then take the $q, \omega$-integral from $a$ to $b$. We obtain the new constraint

$$
\mathcal{K}\left[y_{1}, y_{2}\right]=\int_{a}^{b} \lambda(t) g\left(t, y_{1}(q t+\omega), y_{2}(q t+\omega), D_{q, \omega} y_{1}(t), D_{q, \omega} y_{2}(t)\right) d_{q, \omega} t=0 .
$$


Under the conditions of Theorem 3.3. the solutions $\left(y_{1}, y_{2}\right)$ of the isoperimetric problem (17) and (20) satisfy the Euler-Lagrange equation for the functional

$$
\int_{a}^{b}(f-\tilde{\lambda}(t) g) d_{q, \omega} t
$$

$\tilde{\lambda}(t)=\bar{\lambda} \lambda(t)$ for some constant $\bar{\lambda}$. Since (20) follows from (19), the solutions of problem (17)-(19) satisfy as well the Euler-Lagrange equation for functional (21).

\subsection{Sufficient Conditions}

In this section, we prove sufficient conditions that ensure the existence of minimum (maximum). Similarly to what happens in the classical calculus of variations, some hypotheses of convexity (concavity) are in order.

Definition 3.5. Given a function $f$, we say that $f(\underline{t}, u, v)$ is jointly convex (concave) in $(u, v)$, iff $\partial_{i} f, i=2,3$, exist and are continuous and verify the following condition:

$$
f\left(t, u+u_{1}, v+v_{1}\right)-f(t, u, v) \geq(\leq) \partial_{2} f(t, u, v) u_{1}+\partial_{3} f(t, u, v) v_{1}
$$

for all $(t, u, v),\left(t, u+u_{1}, v+v_{1}\right) \in[a, b]_{q, \omega} \times \mathbb{R}^{2}$.

Theorem 3.5. Let $f(\underline{t}, u, v)$ be jointly convex (concave) in $(u, v)$. If $\tilde{y}$ satisfies condition (8)), then $\tilde{y}$ is a global minimizer (maximizer) to problem (5)-(6).

Proof. We give the proof for the convex case. Since $f$ is jointly convex in $(u, v)$ for any admissible function $\tilde{y}+h$, we have

$$
\begin{aligned}
& \mathcal{L}(\tilde{y}+h)-\mathcal{L}(\tilde{y}) \\
& \quad=\int_{a}^{b}\left[f\left(t, \tilde{y}(q t+\omega)+h(q t+\omega), D_{q, \omega} \tilde{y}(t)+D_{q, \omega} h(t)\right)-f\left(t, \tilde{y}(q t+\omega), D_{q, \omega} \tilde{y}(t)\right)\right] d_{q, \omega} \\
& \quad \geq \int_{a}^{b}\left[\partial_{2} f\left(t, \tilde{y}(q t+\omega), D_{q, \omega} \tilde{y}(t)\right) h(q t+\omega)+\partial_{3} f\left(t, \tilde{y}(q t+\omega), D_{q, \omega} \tilde{y}(t)\right) D_{q, \omega} h(t)\right] d_{q, \omega} .
\end{aligned}
$$

We can now proceed analogously to the proof of Theorem 3.2. As the result we get

$$
\begin{aligned}
\mathcal{L}(\tilde{y}+h)-\mathcal{L}(\tilde{y}) \geq & \left.\partial_{3} f(t, \tilde{y}(q t+\omega), \tilde{y}(t)) h(t)\right|_{t=a} ^{t=b} \\
& +\int_{a}^{b}\left(\partial_{2} f(t, \tilde{y}(q t+\omega), \tilde{y}(t))-D_{q, \omega} \partial_{3} f(t, \tilde{y}(q t+\omega), \tilde{y}(t))\right) h(q t+\omega) d_{q, \omega} t
\end{aligned}
$$

Since $\tilde{y}$ satisfy conditions (8) and $h(a)=h(b)=0$, we obtain $\mathcal{L}(\tilde{y}+h)-\mathcal{L}(\tilde{y}) \geq 0$.

\subsection{Leitmann's Direct Method}

Leitmann's direct method permits to compute global solutions to some problems that are variationally invariant under a family of transformations $[21,26,27,32,33$. It should be mentioned that such invariance transformations are useful not only in connection with Leitmann's method but also to apply Noether's theorem [42,43. Moreover, the invariance transformations are related with the notion of Carathéodory equivalence 22,44].

Recently, it has been noticed by the authors that the invariance transformations, that keep the Lagrangian invariant, do not depend on the time scale [31. This is also true for the generalized Hahn quantum setting that we are considering in this work: given a Lagrangian $f: \mathbb{R} \times \mathbb{R} \times \mathbb{R} \rightarrow \mathbb{R}$, the invariance transformations, that keep it invariant up to a gauge term, are exactly the same if the Lagrangian $f$ is used to define a Hahn quantum functional (5) or a classical functional $\mathcal{L}[y]=\int_{a}^{b} L\left(t, y(t), y^{\prime}(t)\right) d t$ of the calculus of variations. Thus, if the quantum problem we want 
to solve admits an enough rich family of invariance transformations, that keep it invariant up to a gauge term, then one need not to solve a Hahn quantum Euler-Lagrange equation to find its minimizer: instead, we can try to use Leitmann's direct method. The question of how to find the invariance transformations is addressed in [45,46.

Let $\bar{f}:[a, b]_{q, \omega} \times \mathbb{R} \times \mathbb{R} \rightarrow \mathbb{R}$. We assume $(u, v) \rightarrow \bar{f}(t, u, v)$ is a $C^{1}\left(\mathbb{R}^{2}, \mathbb{R}\right)$ function for any $t \in[a, b]_{q, \omega}$, and $\bar{f}\left(\cdot, \bar{y}(\cdot), D_{q, \omega} \bar{y}(\cdot)\right), \partial_{2} \bar{f}\left(\cdot, \bar{y}(\cdot), D_{q, \omega} \bar{y}(\cdot)\right)$, and $\partial_{3} \bar{f}\left(\cdot, \bar{y}(\cdot), D_{q, \omega} \bar{y}(\cdot)\right)$ are continuous in $\omega_{0}$ for any admissible function $\bar{y}(\cdot)$. Consider the integral

$$
\overline{\mathcal{L}}[\bar{y}]=\int_{a}^{b} \bar{f}\left(t, \bar{y}(q t+\omega), D_{q, \omega} \bar{y}(t)\right) d_{q, \omega} t .
$$

Lemma 3.4 (Leitmann's fundamental lemma via Hahn's quantum operator). Let $y=z(t, \bar{y})$ be a transformation having an unique inverse $\bar{y}=\bar{z}(t, y)$ for all $t \in[a, b]_{q, \omega}$, such that there is a one-to-one correspondence

$$
y(t) \Leftrightarrow \bar{y}(t)
$$

for all functions $y \in \mathbb{E}$ satisfying (6) and all functions $\bar{y} \in \mathbb{E}$ satisfying

$$
\bar{y}=\bar{z}(a, \alpha), \quad \bar{y}=\bar{z}(b, \beta) .
$$

If the transformation $y=z(t, \bar{y})$ is such that there exists a function $G:[a, b]_{q, \omega} \times \mathbb{R} \rightarrow \mathbb{R}$ satisfying the functional identity

$$
f\left(t, y(q t+\omega), D_{q, \omega} y(t)\right)-\bar{f}\left(t, \bar{y}(q t+\omega), D_{q, \omega} \bar{y}(t)\right)=D_{q, \omega} G(t, \bar{y}(t)),
$$

then if $\bar{y}^{*}$ yields the extremum of $\overline{\mathcal{L}}$ with $\bar{y}^{*}$ satisfying $(22), y^{*}=z\left(t, \bar{y}^{*}\right)$ yields the extremum of $\mathcal{L}$ for $y^{*}$ satisfying ([6).

Remark 3.3. The functional identity (23) is exactly the definition of variationally invariance when we do not consider transformations of the time variable $t$ (cf. (4) and (5) of [33]). Function $G$ that appears in (23) is sometimes called a gauge term [43.

Proof. The proof is similar in spirit to Leitmann's proof [21,26, 27, 30]. Let $y \in \mathbb{E}$ satisfy (6), and define functions $\bar{y} \in \mathbb{E}$ through the formula $\bar{y}=\bar{z}(t, y), t \in[a, b]_{q, \omega}$. Then $\bar{y} \in \mathbb{E}$ and satisfies (22). Moreover, as a result of (23), it follows that

$$
\begin{aligned}
\mathcal{L}[y]-\overline{\mathcal{L}}[\bar{y}] & =\int_{a}^{b} f\left(t, y(q t+\omega), D_{q, \omega} y(t)\right) d_{q, \omega} t-\int_{a}^{b} \bar{f}\left(t, \bar{y}(q t+\omega), D_{q, \omega} \bar{y}(t)\right) d_{q, \omega} t \\
& =\int_{a}^{b} D_{q, \omega} G(t, \bar{y}(t)) d_{q, \omega} t=G(b, \bar{y}(b))-G(a, \bar{y}(a)) \\
& =G(b, \bar{z}(b, \beta))-G(a, \bar{z}(a, \alpha))
\end{aligned}
$$

from which the desired conclusion follows immediately since the right-hand side of the above equality is a constant, depending only on the fixed-endpoint conditions (6).

Examples 3.2 3.3 and 3.4 in the next section illustrate the applicability of Lemma 3.4. The procedure is as follows: (i) we use the computer algebra package described in [45] and available from the Maple Application Center at http://www . maplesoft.com/applications/view . aspx?SID=4805 to find the transformations that keep the problem of the calculus of variations or optimal control invariant; (ii) we use such invariance transformations to solve the Hahn quantum variational problem by applying Leitmann's fundamental lemma (Lemma 3.4). 


\subsection{Illustrative Examples}

We provide some examples in order to illustrate our main results.

Example 3.1. Let $q, \omega$ be fixed real numbers, and $I$ be a closed interval of $\mathbb{R}$ such that $\omega_{0}, 0,1 \in I$. Consider the problem

$$
\operatorname{minimize} \quad \mathcal{L}[y]=\int_{0}^{1}\left(y(q t+\omega)+\frac{1}{2}\left(D_{q, \omega} y(t)\right)^{2}\right) d_{q, \omega} t
$$

subject to the boundary conditions

$$
y(0)=0, \quad y(1)=1
$$

If $y$ is a local minimizer to problem (24)-(25), then by Theorem 3.2 it satisfies the Euler-Lagrange equation

$$
D_{q, \omega} D_{q, \omega} y(t)=1
$$

for all $t \in\left\{\omega[n]_{q}: n \in \mathbb{N}_{0}\right\} \cup\left\{q^{n}+\omega[n]_{q}: n \in \mathbb{N}_{0}\right\} \cup\left\{\omega_{0}\right\}$. By direct substitution it can be verified that $y(t)=\frac{1}{q+1} t^{2}-\frac{1}{q+1} t$ is a candidate solution to problem (24)-(25).

In next examples we solve quantum variational problems using Leitmann's direct method (see Sect. 3.4).

Example 3.2. Let $q, \omega$, and $a, b(a<b)$ be fixed real numbers, and $I$ be a closed interval of $\mathbb{R}$ such that $\omega_{0} \in I$ and $a, b \in\left\{q^{n} s+[n]_{q, \omega}: n \in \mathbb{N}_{0}\right\} \cup\left\{\omega_{0}\right\}$ for some $s \in I$. Let $\alpha$ and $\beta$ be two given reals, $\alpha \neq \beta$. We consider the following problem:

$$
\begin{gathered}
\operatorname{minimize} \quad \mathcal{L}[y]=\int_{a}^{b}\left(\left(D_{q, \omega} y(t)\right)^{2}+y(q t+\omega)+t D_{q, \omega} y(t)\right) d_{q, \omega} t, \\
y(a)=\alpha, \quad y(b)=\beta .
\end{gathered}
$$

We transform problem (27) into the trivial problem

$$
\operatorname{minimize} \quad \overline{\mathcal{L}}[\bar{y}]=\int_{a}^{b}\left(D_{q, \omega} \bar{y}(t)\right)^{2} d_{q, \omega} t, \quad \bar{y}(a)=0, \quad \bar{y}(b)=0,
$$

which has solution $\bar{y} \equiv 0$. For that we consider the transformation

$$
y(t)=\bar{y}(t)+c t+d, \quad c, d \in \mathbb{R},
$$

where constants $c$ and $d$ will be chosen later. According to the above, we have

$$
D_{q, \omega} y(t)=D_{q, \omega} \bar{y}(t)+c, \quad y(q t+\omega)=\bar{y}(q t+\omega)+c(q t+\omega)+d,
$$

and

$$
\begin{aligned}
\left(D_{q, \omega} y(t)\right)^{2} & +y(q t+\omega)+t D_{q, \omega} y(t) \\
& =\left(D_{q, \omega} \bar{y}(t)\right)^{2}+2 c D_{q, \omega} \bar{y}(t)+c^{2}+\bar{y}(q t+\omega)+c(q t+\omega)+d+t D_{q, \omega} \bar{y}(t)+c t \\
& =\left(D_{q, \omega} \bar{y}(t)\right)^{2}+D_{q, \omega}\left[2 c \bar{y}(t)+t \bar{y}(t)+c t^{2}+\left(c^{2}+d\right) t\right] .
\end{aligned}
$$

In order to obtain the solution to the original problem, it suffices to chose $c$ and $d$ so that

$$
c a+d=\alpha, \quad c b+d=\beta .
$$

Solving the system of equations (28) we obtain $c=\frac{\alpha-\beta}{a-b}$ and $d=\frac{\beta a-b \alpha}{a-b}$. Hence, the global minimizer for problem (27) is

$$
y(t)=\frac{\alpha-\beta}{a-b} t+\frac{\beta a-b \alpha}{a-b} .
$$


Example 3.3. Let $q, \omega$, and $a, b(a<b)$ be fixed real numbers, and $I$ be a closed interval of $\mathbb{R}$ such that $\omega_{0} \in I$ and $a, b \in\left\{q^{n} s+[n]_{q, \omega}: n \in \mathbb{N}_{0}\right\} \cup\left\{\omega_{0}\right\}$ for some $s \in I$. Let $\alpha$ and $\beta$ be two given reals, $\alpha \neq \beta$. We consider the following problem:

$$
\operatorname{minimize} \quad \mathcal{L}[y]=\int_{a}^{b}\left[D_{q, \omega}(y(t) g(t))\right]^{2} d_{q, \omega} t, \quad y(a)=\alpha, \quad y(b)=\beta,
$$

where $g$ does not vanish on the interval $[a, b]_{q, \omega}$. Observe that $\bar{y}(t)=g^{-1}(t)$ minimizes $\mathcal{L}$ with end conditions $\bar{y}(a)=g^{-1}(a)$ and $\bar{y}(b)=g^{-1}(b)$. Let $y(t)=\bar{y}(t)+p(t)$. Then

$$
\left[D_{q, \omega}(y(t) g(t))\right]^{2}=\left[D_{q, \omega}(\bar{y}(t) g(t))\right]^{2}+D_{q, \omega}(p(t) g(t)) D_{q, \omega}(2 \bar{y}(t) g(t)+p(t) g(t)) .
$$

Consequently, if $p(t)=(A t+B) g^{-1}(t)$, where $A$ and $B$ are constants, then (30) is of the form (23), since $D_{q, \omega}(p(t) g(t))$ is constant. Thus, the function

$$
y(t)=(A t+C) g^{-1}(t)
$$

with

$$
A=[\alpha g(a)-\beta g(b)](a-b)^{-1}, \quad C=[a \beta g(b)-b \alpha g(a)](a-b)^{-1},
$$

minimizes (29).

Using the idea of Leitmann, we can also solve quantum optimal control problems defined in terms of Hahn's operators.

Example 3.4. Let $q, \omega$ be real numbers on a closed interval $I$ of $\mathbb{R}$ such that $\omega_{0} \in I$ and $0,1 \in$ $\left\{q^{n} s+[n]_{q, \omega}: n \in \mathbb{N}_{0}\right\} \cup\left\{\omega_{0}\right\}$ for some $s \in I$. Consider the global minimum problem

$$
\left.\operatorname{minimize} \quad \mathcal{L}\left[u_{1}, u_{2}\right]=\int_{0}^{1}\left(\left(u_{1}(t)\right)^{2}+u_{2}(t)\right)^{2}\right) d_{q, \omega} t
$$

subject to the control system

$$
D_{q, \omega} y_{1}(t)=\exp \left(u_{1}(t)\right)+u_{1}(t)+u_{2}(t), \quad D_{q, \omega} y_{2}(t)=u_{2}(t),
$$

and conditions

$$
y_{1}(0)=0, \quad y_{1}(1)=2, \quad y_{2}(0)=0, \quad y_{2}(1)=1, \quad u_{1}(t), u_{2}(t) \in \Omega=[-1,1] .
$$

This example is inspired from [33]. It is worth to mention that due to the constraints on the values of the controls $\left(u_{1}(t), u_{2}(t) \in \Omega=[-1,1]\right)$, a theory based on necessary optimality conditions to solve problem (31)- (33) does not exist at the moment.

We begin noticing that problem (31) - (33) is variationally invariant according to [45] under the one-parameter family of transformations

$$
y_{1}^{s}=y_{1}+s t, \quad y_{2}^{s}=y_{2}+s t, \quad u_{2}^{s}=u_{2}+s \quad\left(t^{s}=t \text { and } u_{1}^{s}=u_{1}\right) .
$$

To prove this, we need to show that both the functional integral $\mathcal{L}$ and the control system stay invariant under the $s$-parameter transformations (34). This is easily seen by direct calculations:

$$
\begin{aligned}
\mathcal{L}^{s}\left[u_{1}^{s}, u_{2}^{s}\right] & =\int_{0}^{1}\left(u_{1}^{s}(t)\right)^{2}+\left(u_{2}^{s}(t)\right)^{2} d_{q, \omega} t \\
& =\int_{0}^{1} u_{1}(t)^{2}+\left(u_{2}(t)+s\right)^{2} d_{q, \omega} t \\
& =\int_{0}^{1}\left(u_{1}(t)^{2}+u_{2}(t)^{2}+D_{q, \omega} t\left[s^{2} t+2 s y_{2}(t)\right]\right) d_{q, \omega} t \\
& =\mathcal{L}\left[u_{1}, u_{2}\right]+s^{2}+2 s
\end{aligned}
$$


We remark that $\mathcal{L}^{s}$ and $\mathcal{L}$ have the same minimizers: adding a constant $s^{2}+2 s$ to the functional $\mathcal{L}$ does not change the minimizer of $\mathcal{L}$. It remains to prove that the control system also remains invariant under transformations (34):

$$
\begin{aligned}
D_{q, \omega}\left(y_{1}^{s}(t)\right) & =D_{q, \omega}\left(y_{1}(t)+s t\right)=D_{q, \omega} y_{1}+s=\exp \left(u_{1}(t)\right)+u_{1}(t)+u_{2}(t)+s \\
& =\exp \left(u_{1}^{s}(t)\right)+u_{1}^{s}(t)+u_{2}^{s}(t), \\
D_{q, \omega}\left(y_{2}^{s}(t)\right) & =D_{q, \omega}\left(y_{2}(t)+s t\right)=D_{q, \omega} y_{2}+s=u_{2}(t)+s \\
& =u_{2}^{s}(t) .
\end{aligned}
$$

Conditions (35) and (36) prove that problem (31)-33) is invariant under the $s$-parameter transformations (34) up to $D_{q, \omega}\left(s^{2} t+2 s y_{2}(t)\right)$. Using the invariance transformations (34), we generalize problem (31)-(33) to a $s$-parameter family of problems, $s \in \mathbb{R}$, which include the original problem for $s=0$ :

$$
\operatorname{minimize} \quad \mathcal{L}^{s}\left[u_{1}, u_{2}\right]=\int_{0}^{1}\left(u_{1}^{s}(t)\right)^{2}+\left(u_{2}^{s}(t)\right)^{2} d_{q, \omega} t
$$

subject to the control system

$$
D_{q, \omega}\left(y_{1}^{s}(t)\right)=\exp \left(u_{1}^{s}(t)\right)+u_{1}^{s}(t)+u_{2}^{s}(t), \quad D_{q, \omega}\left(y_{2}^{s}(t)\right)=u_{2}^{s}(t),
$$

and conditions

$$
\begin{gathered}
y_{1}^{s}(0)=0, \quad y_{1}^{s}(1)=2+s, \quad y_{2}^{s}(0)=0, \quad y_{2}^{s}(1)=1+s, \\
u_{1}^{s}(t) \in[-1,1], \quad u_{2}^{s}(t) \in[-1+s, 1+s] .
\end{gathered}
$$

It is clear that $\mathcal{L}^{s} \geq 0$ and that $\mathcal{L}^{s}=0$ if $u_{1}^{s}(t)=u_{2}^{s}(t) \equiv 0$. The control equations, the boundary conditions and the constraints on the values of the controls imply that $u_{1}^{s}(t)=u_{2}^{s}(t) \equiv 0$ is admissible only if $s=-1: y_{1}^{s=-1}(t)=t, y_{2}^{s=-1}(t) \equiv 0$. Hence, for $s=-1$ the global minimum to $\mathcal{L}^{s}$ is 0 and the minimizing trajectory is given by

$$
\tilde{u}_{1}^{s}(t) \equiv 0, \quad \tilde{u}_{2}^{s}(t) \equiv 0, \quad \tilde{y}_{1}^{s}(t)=t, \quad \tilde{y}_{2}^{s}(t) \equiv 0 .
$$

Since for any $s$ one has by (35) that $\mathcal{L}\left[u_{1}, u_{2}\right]=\mathcal{L}^{s}\left[u_{1}^{s}, u_{2}^{s}\right]-s^{2}-2 s$, we conclude that the global minimum for problem $\mathcal{L}\left[u_{1}, u_{2}\right]$ is 1 . Thus, using the inverse functions of the variational symmetries (34),

$$
u_{1}(t)=u_{1}^{s}(t), \quad u_{2}(t)=u_{2}^{s}(t)-s, \quad y_{1}(t)=y_{1}^{s}(t)-s t, \quad y_{2}(t)=y_{2}^{s}(t)-s t,
$$

and the absolute minimizer for problem (31) $-(33)$ is

$$
\tilde{u}_{1}(t)=0, \quad \tilde{u}_{2}(t)=1, \quad \tilde{y}_{1}(t)=2 t, \quad \tilde{y}_{2}(t)=t .
$$

\subsection{An Application Towards a Quantum Ramsey Model}

As the variables, that are usually considered and observed by the economist, are the outcome of a great number of decisions, taken by different operators at different points of time, it seems natural to look for new kinds of models which are more flexible and realistic. Hahn's approach allows for more complex applications than the discrete or the continuous models. A consumer might have income from work at unequal time intervals and/or make expenditures at unequal time intervals. Therefore, it is possible to obtain more rigorous and more accurate solutions with the approach here proposed.

We discuss the application of the Hahn quantum variational calculus to the Ramsey model, which determines the behavior of saving/consumption as the result of optimal inter-temporal choices by individual households 47. For a complete treatment of the classical Ramsey model we refer the reader to [48]. Before writing the quantum model in terms of the Hahn operators we will present its discrete and continuous versions. The discrete-time Ramsey model is

$$
\max _{\left[W_{t}\right]} \sum_{t=0}^{T-1}(1+p)^{-t} U\left[W_{t}-\frac{W_{t+1}}{1+r}\right], \quad C_{t}=W_{t}-\frac{W_{t+1}}{1+r},
$$


while the continuous Ramsey model is

$$
\max _{W(\cdot)} \int_{0}^{T} e^{-p t} U\left[r W(t)-W^{\prime}(t)\right] d t, \quad C(t)=r W(t)-W^{\prime}(t),
$$

where the quantities are defined as

- $W$ - production function,

- $C$ - consumption,

- $p$ - discount rate,

- $r$ - rate of yield,

- $U$ - instantaneous utility function.

One may assume, due to some constraints of economical nature, that the dynamics do not depend on the usual derivative or the forward difference operator, but on the Hahn quantum difference operator $D_{q, \omega}$. In this condition, one is entitled to assume again that the constraint $C(t)$ has the form

$$
C(t)=-\left[E\left(-r, \frac{t-\omega}{q}\right)\right]^{-1} D_{q, \omega}\left[E\left(-r, \frac{t-\omega}{q}\right) W(t)\right],
$$

where $E(z, \cdot)$ is the $q, \omega$-exponential function defined by

$$
E(z, t):=\prod_{k=0}^{\infty}\left(1+z q^{k}(t(1-q)-\omega)\right)
$$

for $z \in \mathbb{C}$. Several nice properties of the $q, \omega$-exponential function can be found in [17, 18]. By taking the $q, \omega$-derivative of $\left[E\left(-r, \frac{t-\omega}{q}\right) W(t)\right]$ the following is obtained:

$$
\begin{aligned}
C(t)=-\left[E\left(-r, \frac{t-\omega}{q}\right)\right]^{-1}\left[E\left(-r, \frac{t-\omega}{q}\right) D_{q, \omega} W(t)\right. \\
\left.+E\left(-r, \frac{t-\omega}{q}\right) W(q t+\omega) \frac{r\left(1-\frac{1}{q}\right)-r\left(1+r\left(t-\frac{t-\omega}{q}\right)\right)}{\left(1+r\left(t-\frac{t-\omega}{q}\right)\right)(1-r(t(1-q)-\omega))}\right] .
\end{aligned}
$$

The quantum Ramsey model with the Hahn difference operator consists to

$$
\max _{W(\cdot)} \int_{0}^{T} E(-p, t) U\left[W(q t+\omega) \frac{r\left(1+r\left(t-\frac{t-\omega}{q}\right)\right)-r\left(1-\frac{1}{q}\right)}{\left(1+r\left(t-\frac{t-\omega}{q}\right)\right)(1-r(t(1-q)-\omega))}-D_{q, \omega} W(t)\right] d_{q, \omega}
$$

subject to the constraint

$$
C(t)=W(q t+\omega) \frac{r\left(1+r\left(t-\frac{t-\omega}{q}\right)\right)-r\left(1-\frac{1}{q}\right)}{\left(1+r\left(t-\frac{t-\omega}{q}\right)\right)(1-r(t(1-q)-\omega))}-D_{q, \omega} W(t) .
$$

The quantum Euler-Lagrange equation is, by Theorem 3.2, given by

$$
E(-p, t) U^{\prime}[C(t)] \frac{r\left(1+r\left(t-\frac{t-\omega}{q}\right)\right)-r\left(1-\frac{1}{q}\right)}{\left(1+r\left(t-\frac{t-\omega}{q}\right)\right)(1-r(t(1-q)-\omega))}+D_{q, \omega}\left[E(-p, t) U^{\prime}[C(t)]\right]=0 .
$$

Note that for $q \uparrow 1$ and $\omega \downarrow 0$ problem (38)-(39) reduces to (37), and (40) to the classical Ramsey's Euler-Lagrange differential equation. 


\section{Conclusion}

In this paper we consider variational problems in the context of the Hahn quantum calculus. Such variational problems are defined through the Hahn quantum difference operator and the JacksonNörlund integral. The origin of the Hahn quantum difference operator dates back to a 1949 paper of W. Hahn [6] where it was introduced to unify, in a limiting sense, the Jackson $q$-difference derivative and the forward difference. For both of these latter two quantum difference operators, variational problems have been studied previously. The forward difference problems were studied at least as early as 1937 by T. Fort [20] and for the $q$-difference by G. Bangerezako in 2004 [2]. In both of these works the authors discuss necessary conditions for optimality and obtain the analogue of the classical Euler-Lagrange equation, as well as other classical results. The goal of the present paper is to provide extensions of the previous results for the more general Hahn quantum difference operator.

Another related course of study is that of the notion of a time scale. The origins of this idea dates back to the late 1980's when S. Hilger introduced this notion in his Ph.D. thesis (directed by B. Aulbach) and showed how to unify continuous time and discrete time dynamical systems 49 . Since this important result, the literature has exploded with papers and books on time scales in which many known results for ordinary differential equations and difference equations have been combined and extended [31, 39, 47. The classical results of the calculus of variations have been extended to times scales by M. Bohner in 2004 [39]. However, the Euler-Lagrange equation here obtained is not comparable with that of [39]. Indeed, the Hahn quantum calculus is not covered by the Hilger time scale theory. This is well explained, for example, in the $2009 \mathrm{Ph} . \mathrm{D}$. thesis of Aldwoah [17] (see also [35]). Here we just note the following: if in Bohner's paper [39] one chooses the time scale to be the $q$-scale $\mathbb{T}:=\left\{q^{n}: n \in \mathbb{Z}\right\}$, then the expression of the delta-derivative coincides with the expression of the Jackson $q$-difference derivative. However, they are not the same. There is an important distinction: the Jackson $q$-difference derivative is defined in the set of real numbers while the time-scale derivative is only defined in a subset $\mathbb{T}$ of the real numbers. One more difference, between the Hahn calculus we use in this paper and the time scale theory, is the following: the delta integral satisfies all the usual properties of the Riemann integral while this is not the case with the Jackson-Nörlund integral: the inequality (44) is not always true for the Jackson-Nörlund integral.

The main advantage of our results is that they are able to deal with nondifferentiable functions, even discontinuous functions, that are important in physical systems. Quantum derivatives and integrals play a leading role in the understanding of complex physical systems. For example, in 1992 Nottale introduced the theory of scale-relativity without the hypothesis of space-time differentiability [50. A rigorous mathematical foundation to Nottale's scale-relativity theory is nowadays given by means of a quantum calculus [1,4. We remark that results in Bohner's paper [39] are not able to deal with such nondifferentiable functions. Variational problems in 39] are formulated for functions that are delta-differentiable. It is well known that delta-differentiable functions are necessarily continuous. This is not the case in our context: see Example 2.2, where a discontinuous function is $q, \omega$-differentiable in all the real interval $[-1,1]$.

We believe that the obtained results are of interest in Economics. Economists model time as continuous or discrete. The kind of "time" (continuous or discrete) to be used in the construction of dynamic models is a moot question. Although individual economic decisions are generally made at discrete time intervals, it is difficult to believe that they are perfectly synchronized as postulated by discrete models. The usual assumption that the economic activity takes place continuously, is a convenient abstraction in many applications. In others, such as the ones studied in financial-market equilibrium, the assumption of continuous trading corresponds closely to reality. We believe that our Hahn's approach helps to bridge the gap between two families of models: continuous and discrete. We trust that the field here initiated will prove fruitful for further research. 


\section{References}

1. Almeida, R., Torres, D.F.M.: Hölderian variational problems subject to integral constraints. J. Math. Anal. Appl. 359(2), 674-681 (2009) arXiv:0807.3076

2. Bangerezako, G.: Variational $q$-calculus. J. Math. Anal. Appl. 289(2), 650-665 (2004)

3. Bangerezako, G.: Variational calculus on $q$-nonuniform lattices. J. Math. Anal. Appl. 306(1), 161-179 (2005)

4. Cresson, J., Frederico, G.S.F., Torres, D.F.M.: Constants of motion for non-differentiable quantum variational problems. Topol. Methods Nonlinear Anal. 33(2), 217-231 (2009) arXiv:0805.0720

5. Kac, V., Cheung, P.: Quantum calculus. Springer, New York (2002)

6. Hahn, W.: Über orthogonalpolynome, die $q$-differenzenlgleichungen genügen. Math. Nachr. 2, $4-34(1949)$

7. Gasper, G., Rahman, M.: Basic hypergeometric series, Second edition. Cambridge Univ. Press, Cambridge (2004)

8. Jackson, F.H.: Basic integration. Quart. J. Math., Oxford Ser. (2) 2, 1-16 (1951)

9. Bird, M.T.: On generalizations of sum formulas of the Euler-Maclaurin type. Amer. J. Math. 58(3), 487-503 (1936)

10. Jagerman, D.L.: Difference equations with applications to queues. Dekker, New York (2000)

11. Jordan, C.: Calculus of finite differences, Third Edition. Introduction by Carver, H.C. Chelsea, New York (1965).

12. Álvarez-Nodarse, R.: On characterizations of classical polynomials, J. Comput. Appl. Math. 196(1), 320-337 (2006)

13. Costas-Santos, R.S., Marcellán, F.: Second structure relation for $q$-semiclassical polynomials of the Hahn tableau. J. Math. Anal. Appl., 329(1), 206-228 (2007)

14. Dobrogowska, A., Odzijewicz, A.: Second order $q$-difference equations solvable by factorization method. J. Comput. Appl. Math. 193(1), 319-346 (2006)

15. Kwon, K.H., Lee, D.W., Park, S.B., Yoo, B.H.: Hahn class orthogonal polynomials. Kyungpook Math. J. 38(2), 259-281 (1998)

16. Petronilho, J.: Generic formulas for the values at the singular points of some special monic classical $H_{q, \omega}$-orthogonal polynomials. J. Comput. Appl. Math. 205(1), 314-324 (2007)

17. Aldwoah, K.A.: Generalized time scales and associated difference equations. Ph.D. Thesis, Cairo University (2009)

18. Annaby, M.H., Hamza, A.E., Aldwoah, K.A.: Hahn difference operator and associated Jackson-Nörlund integrals. Preprint (2009)

19. Kelley, W.G., Peterson, A.C.: Difference equations, Second edition. Harcourt/Academic Press, San Diego, CA (2001)

20. Fort, T.: The calculus of variations applied to Nörlund's sum, Bull. Amer. Math. Soc. 43(12), 885-887 (1937)

21. Leitmann, G.: A note on absolute extrema of certain integrals. Internat. J. Non-Linear Mech. 2, 55-59 (1967) 
22. Carlson, D.A.: An observation on two methods of obtaining solutions to variational problems. J. Optim. Theory Appl. 114(2), 345-361 (2002)

23. Carlson, D.A., Leitmann, G.: Coordinate transformation method for the extremization of multiple integrals. J. Optim. Theory Appl. 127(3), 523-533 (2005)

24. Carlson, D.A., Leitmann, G.: A direct method for open-loop dynamic games for affine control systems. In: Dynamic games: theory and applications, pp. 37-55. Springer, New York (2005)

25. Carlson, D.A., Leitmann, G.: Fields of extremals and sufficient conditions for the simplest problem of the calculus of variations. J. Global Optim. 40(1-3), 41-50 (2008)

26. Leitmann, G.: On a class of direct optimization problems. J. Optim. Theory Appl. 108(3), 467-481 (2001)

27. Leitmann, G.: Some extensions to a direct optimization method. J. Optim. Theory Appl. 111(1), 1-6 (2001)

28. Leitmann, G.: On a method of direct optimization. Vychisl. Tekhnol. 7, 63-67 (2002)

29. Leitmann, G.: A direct method of optimization and its application to a class of differential games. Cubo Mat. Educ. 5(3), 219-228 (2003)

30. Leitmann, G.: A direct method of optimization and its application to a class of differential games. Dyn. Contin. Discrete Impuls. Syst. Ser. A Math. Anal. 11(2-3), 191-204 (2004)

31. Malinowska, A.B., Torres, D.F.M.: Leitmann's direct method of optimization for absolute extrema of certain problems of the calculus of variations on time scales. Appl. Math. Comput. (2010), in press. DOI: 10.1016/j.amc.2010.01.015 arXiv:1001.1455

32. Silva, C.J., Torres, D.F.M.: Absolute extrema of invariant optimal control problems. Commun. Appl. Anal. 10(4), 503-515 (2006) arXiv:math/0608381

33. Torres, D.F.M., Leitmann, G.: Contrasting two transformation-based methods for obtaining absolute extrema. J. Optim. Theory Appl. 137(1), 53-59 (2008) arXiv:0704.0473

34. Wagener, F.O.O.: On the Leitmann equivalent problem approach. J. Optim. Theory Appl. 142(1), 229-242 (2009)

35. Aldwoah, K.A., Hamza, A.E.: Difference time scales, Int. J. Math. Stat. 9(A11), 106-125 (2011)

36. Jackson, F.H.: On q-definite integrals. Quart. J. Pure and Appl. Math. 41, 193-203 (1910)

37. Fort, T.: Finite Differences and Difference Equations in the Real Domain. Oxford, at the Clarendon Press (1948)

38. Nörlund, N.: Vorlesungen über Differencenrechnung. Springer Verlag, Berlin (1924)

39. Bohner, M.: Calculus of variations on time scales. Dynam. Systems Appl. 13(3-4), 339-349 (2004)

40. van Brunt, B.: The calculus of variations. Springer, New York (2004)

41. Arutyunov, A.V.: Optimality conditions-Abnormal and degenerate problems. Kluwer Acad. Publ., Dordrecht (2000)

42. Torres, D.F.M.: On the Noether theorem for optimal control. Eur. J. Control 8(1), 56-63 (2002)

43. Torres, D.F.M.: Proper extensions of Noether's symmetry theorem for nonsmooth extremals of the calculus of variations. Commun. Pure Appl. Anal. 3(3), 491-500 (2004) 
44. Torres, D.F.M.: Carathéodory equivalence Noether theorems, and Tonelli full-regularity in the calculus of variations and optimal control. J. Math. Sci. (N. Y.) 120(1), 1032-1050 (2004) arXiv:math/0206230

45. Gouveia, P.D.F., Torres, D.F.M.: Automatic computation of conservation laws in the calculus of variations and optimal control. Comput. Methods Appl. Math. 5(4), 387-409 (2005) arXiv:math/0509140

46. Gouveia, P.D.F., Torres, D.F.M., Rocha, E.A.M.: Symbolic computation of variational symmetries in optimal control. Control Cybernet. 35(4), 831-849 (2006) arXiv:math/0604072

47. Atici, F.M., McMahan, C.S.: A comparison in the theory of calculus of variations on time scales with an application to the Ramsey Model. Nonlinear Dyn. Syst. Theory 9(1), 1-10 (2009)

48. Barro, R.J., Sala-i-Martin, X.: Economic growth. MIT Press, Cambridge (1999)

49. Aulbach, B., Hilger, S.: A unified approach to continuous and discrete dynamics, in Qualitative theory of differential equations (Szeged, 1988), 37-56, Colloq. Math. Soc. János Bolyai, 53, North-Holland, Amsterdam (1990)

50. Nottale, L.: The theory of scale relativity, Internat. J. Modern Phys. A 7(20), 4899-4936 (1992) 